\title{
Bir Parametreli Eliptik Homotetik Düzlemsel Hareketler
}

\author{
Ayşe Zeynep Azak* ${ }^{*}$ \\ Sakarya Üniversitesi, Matematik ve Fen Bilimleri Eğitimi Bölümü, 54300 Sakarya, Türkiye \\ Geliş / Received: 02/12/2018, Kabul / Accepted: 13/05/2019 \\ Bu makalede bir parametreli eliptik homotetik düzlemsel hareket için türev formülleri elde edildi. Daha sonra \\ bu hareketin mutlak, izafi ve sürüklenme hız vektörleri ve bu vektörler arasındaki ilişkiyi ifade eden teorem \\ verildi. Ayrıca bir parametreli eliptik homotetik hareket için pol noktaları ve kanonik izafi sistem tanıtıldı. Son \\ olarakta $a_{1}, a_{2}$ ve homotetik sabitin 1 olması durumlarında bazı önemli sonuçlar bulundu.
}

\section{$\ddot{O} \mathbf{z}$}

Anahtar Kelimeler: Bir parametreli hareket, eliptik hareket, homotetik hareket

\section{One Parameter Elliptical Homothetic Planar Motions}

\begin{abstract}
In this paper derivative formulas have been obtained for the one parameter elliptical homothetic planar motion. Then, absolute, relative and sliding velocities of this motion and a theorem which states the relationship among these velocities have been given. Also, pole points and the canonical relative system have been introduced for the one-parameter elliptical homothetic motion. Finally, some important results have been found in the case of $a_{1}, a_{2}$ and the homothetic scale being 1 .
\end{abstract}

Keywords: One parameter motion, elliptical motion, homothetic motion

\section{Giriş}

Hareket, fiziksel olarak bir katı cismin belli bir referans noktasına göre sürekli olarak yer değiştirmesi olayıdır. Katı cisimlerin hareketlerinde düzlem hareketi önemli bir yer tutar. $\mathrm{Bu}$ yüzden, ilk olarak bir parametreli düzlemsel hareketler Müller tarafından tanıtıldı ve mutlak, izafi, sürüklenme hızları ve ivmeleri arasındaki bağıntılar Öklid düzlemi $E^{2}$ de elde edildi. Ayrıca yörünge eğrilerinin eğrilikleri arasındaki ilişkiyi veren Euler-Savary formülü verildi (Müller 1963). Blaschke ve Müller tarafindan kompleks sayılar kullanılarak düzlemsel hareket tanıtıldı (Blaschke ve Müller, 1956). Kompleks düzlemde hareketli koordinat sistemi yardımıyla bir parametreli hareket için kompleks hızlar arasındaki bağıntılar ve pol noktaları elde edildi (Tutar vd. 2001). Böylece kompleks düzlemde kanonik izafi sistemi ve Euler-Savary formülü ifade edildi (Masal vd. 2010).

Hareketler uzaklığı koruyan dönüşümlerle verilirler. Homotetik hareketler ise uzaklıkları aynı oranda değiştirirken, açıları koruyan dönüşümlerle verilirler. Dolayısıyla da hareketler, homotetik hareketlerin özel bir halidir. Olcaylar, homotetik dönüşümler yardımıyla bir düzlemin diğer düzlem üzerindeki homotetik hareketini tanımladı (Olcaylar 1956). Hacisalihoğlu tarafindan ise n-boyutlu Öklid uzayında homotetik hareketleri tanımlandi ve mutlak, izafi, sürüklenme hızları elde edildi. Ayrıca 
hareketin $t$ anındaki ani dönme pol noktaları bulundu. $\mathrm{Bu}$ homotetik hareket altında pol eğrilerinin birbirleri üzerinde kayarak, yuvarlandığını gösterildi. Son olarak da Olcaylar tarafından tanımlanan düzlemsel ve üç boyutlu homotetik hareketler için elde edilen özelliklerin genellendiği ifade edildi (Hacısalihoğlu 1971).

Kuruoğlu, Tutar ve Düldül tarafından bir parametreli kompleks düzlemsel homotetik hareketler tanımlandı ve pol noktaları elde edildi (Kuruoğlu vd. 2001). Daha sonra Masal, Güngör ve Ersoy tarafindan bir parametreli kompleks düzlemsel homotetik hareket için kanonik izafi sistemi oluşturularak, Euler-Savary formülü ifade edildi. $h=1$ olmas 1 durumunda Euler-Savary formülünün, bir parametreli kompleks düzlemsel hareket için Euler-Savary formülüne karşılık geldiği sonucu verildi (Masal vd. 2014).

Farklı eksen uzunluklarına sahip bir elipsoidin eliptik iç çarpım ve eliptik vektörel çarpım yardımıyla eliptik dönmelerinin genellemesi Özdemir tarafından verildi. Eliptik ortogonal matris, eliptik simetrik ve anti-simetrik matrisler tanımlandı. Ayrıca, eliptik dönme matrisleri kullanılarak elipsoid üzerindeki bir noktanın hareketi çalışıldı (Özdemir 2016). Böylece bir parametreli eliptik düzlemsel hareketler için mutlak, izafi, sürüklenme hız vektörleri tanımlandı. Ayrıca bu vektörler arasındaki ilişki ifade edildi. Hareketin pol noktaları bulundu. Kanonik izafi sistemi tanımland1. Son olarak da $a_{1}=1$ ve $a_{2}=1$ olması durumunda ispat edilen teoremlerin bir parametreli Öklidyen düzlemsel hareketleri için elde edilen teoremlere karş1lık geldiği görüldü (Azak 2018).

\section{Materyal ve Metot}

$\mathrm{Bu}$ bölümde eliptik iç çarpım, eliptik norm, eliptik anlamda vektörlerin ortogonal ve ortonormal olmas1, eliptik ortogonal matris ve son olarak eliptik dönme matrisi kavramlarına yer verilecektir.

Orijin merkezli, farklı eksen uzunluklarına sahip bir elipsin standart denklemi

$$
\frac{x^{2}}{a^{2}}+\frac{y^{2}}{b^{2}}=1
$$

şeklinde verilir. $\mathrm{Bu}$ durumda orijin ile elips üzerinden aldığımız herhangi bir nokta arasındaki uzaklığı değiştirmeyecek şekilde özel bir iç çarpımı bu elips yardımıyla tanımlayabiliriz.

$$
\vec{x}=\left(x_{1}, x_{2}\right), \vec{y}=\left(y_{1}, y_{2}\right) \in R^{2}
$$

vektörlerinin eliptik iç çarpımı ya da $B$-iç çarpımı

$$
B(\vec{x}, \vec{y})=a_{1} x_{1} y_{1}+a_{2} x_{2} y_{2}
$$

olarak tanımlanır. Burada $a_{1}=\frac{1}{a^{2}}$ ve $a_{2}=\frac{1}{b^{2}}$ $a$ ile $b$ sıfirdan farklı, reel sayılardır. Bu iç çarpım pozitif tanımlı ve dejenere olmayan bir iç çarpımdır. Böylece eliptik iç çarpım ile ilişkilendirilen reel vektör uzayı $R^{2}, R_{a_{1}, a_{2}}^{2}$ ile gösterilecektir.

Bir $\quad \vec{x} \in R_{a_{1}, a_{2}}^{2}$ vektörünün eliptik anlamda normu

$$
\|\vec{u}\|_{B}=\sqrt{B(\vec{x}, \vec{x})}
$$

olarak tanımlanır. Eğer $\vec{x}$ ve $\vec{y}$ vektörleri için $B(\vec{x}, \vec{y})=0$ oluyorsa bu vektörlere $B$ ortogonal ya da eliptik ortogonal vektörler denir. Aynı zamanda, bu vektörlerin normları 1 oluyorsa bu vektörler eliptik ortonormal vektörler olarak adlandırılırlar. 
Her $\quad \vec{x}, \vec{y} \in R^{2} \quad$ için $\quad B(\overrightarrow{R x}, \overrightarrow{R y})=B(\vec{x}, \vec{y})$ eşitliğini sağlayan $R$ matrisine $B$-ortogonal matris denir. $B$-ortogonal matrislerin kümesi $O B(n)$ ile gösterilsin. Bu durumda

$O B(n)=\left\{R \in R^{2 \times 2}: R^{t} \Omega R=\Omega, \operatorname{det} R= \pm 1\right\}$

olarak verilir. Burada $\Omega$ eliptik iç çarpımla ilişkili matristir ve

$$
\Omega=\left[\begin{array}{ll}
a_{1} & 0 \\
0 & a_{2}
\end{array}\right]
$$

şeklindedir.

$R_{a_{1}, a_{2}}^{2} \quad$ uzayındaki eliptik dönme matrisi

$$
R_{\theta}^{B}=\left[\begin{array}{cc}
\cos \theta & \frac{\sqrt{a_{2}}}{\sqrt{a_{1}}} \sin \theta \\
-\frac{\sqrt{a_{1}}}{\sqrt{a_{2}}} \sin \theta & \cos \theta
\end{array}\right]
$$

olarak tanımlanır. Burada $a_{1}, a_{2} \in R^{+}$dır (Özdemir 2016).

\section{Bir Parametreli Eliptik Homotetik Düzlem Hareketi Bulgular}

$E$ ve $E^{\prime}$ eliptik düzlemlerine göre hareket eden bir $E_{1}$ düzlemini göz önüne alalım. $\mathrm{Bu}$ düzlemlerin ilki hareketli, ikincisi ise sabit olsun. $E_{1}$ hareketli eliptik düzleminin $E$ ve $E^{\prime}$ eliptik düzlemlerine göre hareketini incelemek için $\left\{B ; \vec{h}_{1}, \vec{h}_{2}\right\},\left\{O ; \vec{e}_{1}, \vec{e}_{2}\right\} \quad$ ve $\left\{O^{\prime} ; \vec{e}_{1}^{\prime}, \vec{e}_{2}^{\prime}\right\}$ koordinat sistemlerini temsilci olarak kabul edelim.

Burada, vektörlerin eliptik iç çarpımları sırasıyla aşağıdaki şekilde verilebilir: $B\left(\vec{e}_{i}, \vec{e}_{j}\right)=\left\{\begin{array}{lll}a_{i}, & \text { eğer } & i=j \\ 0, & \text { eğer } & i \neq j\end{array}\right.$
$B\left(\vec{e}_{i}^{\prime}, \vec{e}_{j}^{\prime}\right)=\left\{\begin{array}{lll}a_{i}, & \text { eğer } i=j \\ 0, & \text { eğer } i \neq j .\end{array}\right.$

$\theta$ ve $\theta^{\prime}$ sirasiyla $E_{1} / E$ ve $E_{1} / E^{\prime}$ bir parametreli eliptik düzlem hareketlerinin eliptik dönme açılarını göstermek üzere bir an için koordinat sistemlerinin başlangıç noktaları olan $O, B$ ve $O^{\prime}, B$ noktalarının çakıştığı düşünülürse, aşağıdaki bağıntılar verilebilir:

$\overrightarrow{h_{1}}=\cos \theta \overrightarrow{e_{1}}+\frac{\sqrt{a_{2}}}{\sqrt{a_{1}}} \sin \theta \overrightarrow{e_{2}}$

$\overrightarrow{h_{2}}=-\frac{\sqrt{a_{1}}}{\sqrt{a_{2}}} \sin \theta \overrightarrow{e_{1}}+\cos \theta \overrightarrow{e_{2}}$

ve

$\overrightarrow{h_{1}}=\cos \theta^{\prime} \overrightarrow{e_{1}^{\prime}}+\frac{\sqrt{a_{2}}}{\sqrt{a_{1}}} \sin \theta^{\prime} \overrightarrow{e_{2}^{\prime}}$

$\overrightarrow{h_{2}}=-\frac{\sqrt{a_{1}}}{\sqrt{a_{2}}} \sin \theta^{\prime} \overrightarrow{e_{1}^{\prime}}+\cos \theta^{\prime} \overrightarrow{e_{2}^{\prime}}$.

$E_{1}$ hareketli düzlemi üzerinde koordinatları $x_{1}, x_{2}$ olan bir $X$ noktasi alalım. $\overrightarrow{B X}, \overrightarrow{O B}, \overrightarrow{O^{\prime} B}$ vektörlerini sırasıyla

$\overrightarrow{\tilde{x}}=x_{1} \overrightarrow{h_{1}}+x_{2} \overrightarrow{h_{2}}$,

$\vec{b}=b_{1} \overrightarrow{h_{1}}+b_{2} \overrightarrow{h_{2}}$,

$\overrightarrow{b^{\prime}}=b_{1}^{\prime} \overrightarrow{h_{1}}+b_{2}^{\prime} \overrightarrow{h_{2}}$

olarak ifade edelim. (3) ve (5) denklemlerinin diferansiyeli alınır ve gerekli düzenlemeler yapılırsa $E_{1} / E$ bir parametreli hareketi için aşağıdaki türev denklemleri elde edilir. 
$d \overrightarrow{h_{1}}=\frac{\sqrt{a_{2}}}{\sqrt{a_{1}}} d \theta \overrightarrow{h_{2}}$
$d \overrightarrow{h_{2}}=-\frac{\sqrt{a_{1}}}{\sqrt{a_{2}}} d \theta \overrightarrow{h_{1}}$
$d \vec{b}=\left(d b_{1}-\frac{\sqrt{a_{1}}}{\sqrt{a_{2}}} b_{2} d \theta\right) \overrightarrow{h_{1}}+\left(d b_{2}+\frac{\sqrt{a_{2}}}{\sqrt{a_{1}}} b_{1} d \theta\right) \overrightarrow{h_{2}}$.

$$
\begin{aligned}
& d \overrightarrow{h_{1}}=\frac{\sqrt{a_{2}}}{\sqrt{a_{1}}} \lambda \overrightarrow{h_{2}}, \\
& d \overrightarrow{h_{2}}=-\frac{\sqrt{a_{1}}}{\sqrt{a_{2}}} \lambda \overrightarrow{h_{1}}, \\
& d \vec{b}=\sigma_{1} \overrightarrow{h_{1}}+\sigma_{2} \overrightarrow{h_{2}}
\end{aligned}
$$

Benzer şekilde, (4) ve (5) denklemlerinin diferansiyeli alınır ve gerekli düzenlemeler yapilırsa da $E_{1} / E^{\prime}$ bir parametreli hareketi için aşağıdaki türev denklemleri bulunur.

$$
\begin{aligned}
d^{\prime} \overrightarrow{h_{1}} & =\frac{\sqrt{a_{2}}}{\sqrt{a_{1}}} d \theta^{\prime} \overrightarrow{h_{2}} \\
d^{\prime} \overrightarrow{h_{2}} & =-\frac{\sqrt{a_{1}}}{\sqrt{a_{2}}} d \theta^{\prime} \overrightarrow{h_{1}} \\
d^{\prime} \vec{b} & =\left(d b_{1}^{\prime}-\frac{\sqrt{a_{1}}}{\sqrt{a_{2}}} b_{2}^{\prime} d \theta^{\prime}\right) \overrightarrow{h_{1}} \\
& +\left(d b_{2}^{\prime}+\frac{\sqrt{a_{2}}}{\sqrt{a_{1}}} b_{1}^{\prime} d \theta^{\prime}\right) \overrightarrow{h_{2}} .
\end{aligned}
$$

(6) ve (7) denklemleri kısaca ifade etmek amaciyla

$$
\begin{aligned}
& \lambda=d \theta, \quad \sigma_{1}=d b_{1}-\frac{\sqrt{a_{1}}}{\sqrt{a_{2}}} b_{2} d \theta \\
& \sigma_{2}=d b_{2}+\frac{\sqrt{a_{2}}}{\sqrt{a_{1}}} b_{1} d \theta \\
& \lambda^{\prime}=d \theta^{\prime}, \quad \sigma_{1}^{\prime}=d b_{1}^{\prime}-\frac{\sqrt{a_{1}}}{\sqrt{a_{2}}} b_{2}^{\prime} d \theta^{\prime} \\
& \sigma_{2}^{\prime}=d b_{2}^{\prime}+\frac{\sqrt{a_{2}}}{\sqrt{a_{1}}} b_{1}^{\prime} d \theta^{\prime}
\end{aligned}
$$

olarak alınırsa, $E_{1} / E$ ve $E_{1} / E^{\prime}$ hareketlerinin sırasiyla türev denklemleri

$$
\begin{aligned}
& d^{\prime} \overrightarrow{h_{1}}=\frac{\sqrt{a_{2}}}{\sqrt{a_{1}}} \lambda^{\prime} \overrightarrow{h_{2}}, \\
& d^{\prime} \overrightarrow{h_{2}}=-\frac{\sqrt{a_{1}}}{\sqrt{a_{2}}} \lambda^{\prime} \overrightarrow{h_{1}}, \\
& d^{\prime} \vec{b}=\sigma_{1}^{\prime} \overrightarrow{h_{1}}+\sigma_{2}^{\prime} \overrightarrow{h_{2}}
\end{aligned}
$$

olarak verilir. Burada $\sigma_{1}, \sigma_{2}, \sigma_{1}^{\prime}, \sigma_{2}{ }^{\prime}$ hareketin Pfaff formlarıdır.

$X$ noktasının herhangi bir $t$ anında

$\vec{x}=\vec{b}+h \overrightarrow{\tilde{x}}=\left(b_{1}+h x_{1}\right) \overrightarrow{h_{1}}+\left(b_{2}+h x_{2}\right) \overrightarrow{h_{2}}$

ve

$$
\overrightarrow{x^{\prime}}=\overrightarrow{b^{\prime}}+h \overrightarrow{\tilde{x}}=\left(b_{1}^{\prime}+h x_{1}\right) \overrightarrow{h_{1}}+\left(b_{2}^{\prime}+h x_{2}\right) \overrightarrow{h_{2}}
$$

denklemlerini yazabiliriz. Burada $h=h(t)$ hareketin homoteti sabiti iken $\vec{x}$ ve $\overrightarrow{x^{\prime}}$ de $X$ noktasının, $E$ ve $E^{\prime}$ eliptik düzlemlerinin koordinat sistemlerine göre konum vektörleridir. Hareketin hızları (10) ve (11) denklemlerinin diferensiyelleri alınarak bulunabilir. O zaman, (10) denkleminin diferensiyeli aşağıdaki şekilde elde edilir.

$$
\begin{aligned}
d \vec{x} & =\left(d h x_{1}+h d x_{1}+\sigma_{1}-h \frac{\sqrt{a_{1}}}{\sqrt{a_{2}}} x_{2} \lambda\right) \overrightarrow{h_{1}} \\
& +\left(d h x_{2}+h d x_{2}+\sigma_{2}+h \frac{\sqrt{a_{2}}}{\sqrt{a_{1}}} x_{1} \lambda\right) \overrightarrow{h_{2}} .
\end{aligned}
$$


Böylece, $\quad X$ noktasının $E$ hareketli düzlemine göre hızını ifade eden izafi hız vektörü

$$
\overrightarrow{V_{r}}=\frac{d \vec{x}}{d t}
$$

dir. $X$ noktasının $E^{\prime}$ ye göre diferansiyeli ise

$$
\begin{aligned}
d^{\prime} \vec{x} & =\left(d h x_{1}+h d x_{1}+\sigma_{1}^{\prime}-h \frac{\sqrt{a_{1}}}{\sqrt{a_{2}}} x_{2} \lambda^{\prime}\right) \overrightarrow{h_{1}} \\
& +\left(d h x_{2}+h d x_{2}+\sigma_{2}^{\prime}+h \frac{\sqrt{a_{2}}}{\sqrt{a_{1}}} x_{1} \lambda^{\prime}\right) \overrightarrow{h_{2}}
\end{aligned}
$$

olur. O halde, $X$ noktasinın $E^{\prime}$ sabit düzlemine göre hızı olan mutlak hız vektörü

$$
\overrightarrow{V_{a}}=\frac{d^{\prime} \vec{x}}{d t}
$$

olarak tanımlanır.

Eğer $\vec{V}_{r}=0$ olursa $X$ noktası $E$ düzleminde sabit kalır. (12) denklemi göz önüne alınırsa, $X$ noktasının $E$ hareketli düzleminde sabit kalma koşulları

$$
\begin{aligned}
& h d x_{1}=-d h x_{1}-\sigma_{1}+h \frac{\sqrt{a_{1}}}{\sqrt{a_{2}}} x_{2} \lambda, \\
& h d x_{2}=-d h x_{2}-\sigma_{2}-h \frac{\sqrt{a_{2}}}{\sqrt{a_{1}}} x_{1} \lambda
\end{aligned}
$$

olarak elde edilir. Benzer şekilde eğer $\overrightarrow{V_{a}}=0$ olursa, $X$ noktası $E^{\prime}$ düzleminde sabit kalır. Böylece, (13) denkleminden $X$ noktasının $E^{\prime}$ sabit düzleminde sabit kalma koşulları da

$$
\begin{aligned}
& h d x_{1}=-d h x_{1}-\sigma_{1}^{\prime}+h \frac{\sqrt{a_{1}}}{\sqrt{a_{2}}} x_{2} \lambda^{\prime}, \\
& h d x_{2}=-d h x_{2}-\sigma_{2}^{\prime}-h \frac{\sqrt{a_{2}}}{\sqrt{a_{1}}} x_{1} \lambda^{\prime}
\end{aligned}
$$

olarak bulunur. (14) denklemi denkleminde yerine yazılırsa,

$$
\begin{aligned}
d_{f} \vec{x} & =\left[\left(\sigma_{1}^{\prime}-\sigma_{1}\right)-h x_{2} \frac{\sqrt{a_{1}}}{\sqrt{a_{2}}}\left(\lambda^{\prime}-\lambda\right)\right] \overrightarrow{h_{1}} \\
& +\left[\left(\sigma_{2}^{\prime}-\sigma_{2}\right)+h x_{1} \frac{\sqrt{a_{2}}}{\sqrt{a_{1}}}\left(\lambda^{\prime}-\lambda\right)\right] \overrightarrow{h_{2}} .
\end{aligned}
$$

elde edilir. Burada $X$ noktasının sürüklenme hız vektörü

$$
\overrightarrow{V_{f}}=\frac{d_{f} \vec{x}}{d t}
$$

ile verilir.

Böylece hızlar arasındaki ilişkiyi gösteren aşağıdaki teorem verilebilir.

Teorem 1. $X$ noktası $E_{1}$ hareketli düzlemi üzerinde bir nokta ve $\overrightarrow{V_{r}}, \overrightarrow{V_{a}}, \overrightarrow{V_{f}}$ vektörleri de $X$ in bir parametreli eliptik homotetik hareket altındaki izafi, mutlak ve sürüklenme hızları olsun. Hızlar arasında aşağıdaki şekilde bir bağıntı mevcuttur:

$$
\overrightarrow{V_{a}}=\overrightarrow{V_{r}}+\overrightarrow{V_{f}} .
$$

İspat: (12) ve (16) eşitlikleri taraf tarafa toplandiğında

$$
d^{\prime} \vec{x}=d \vec{x}+d_{f} \vec{x}
$$

eşitliğinin elde edileceği kolaylıkla görülebilir. Böylece $t$ zaman parametresine göre değişim göz önüne alınırsa

$$
\overrightarrow{V_{a}}=\overrightarrow{V_{r}}+\overrightarrow{V_{f}} .
$$

sonucuna ulaşılır.

Şimdi de hareketin pol noktaları ile ilgili teoremi verelim.

Sonuç 1. $a_{1}=1, a_{2}=1$ olması durumunda izafi, mutlak ve sürüklenme hız vektörleri, sirasiyla, 


$$
\begin{aligned}
d \vec{x} & =\left(d h x_{1}+h d x_{1}+\sigma_{1}-h x_{2} \lambda\right) \overrightarrow{h_{1}} \\
& +\left(d h x_{2}+h d x_{2}+\sigma_{2}+h x_{1} \lambda\right) \overrightarrow{h_{2}} \\
d^{\prime} \vec{x} & =\left(d h x_{1}+h d x_{1}+\sigma_{1}^{\prime}-h x_{2} \lambda^{\prime}\right) \overrightarrow{h_{1}} \\
& +\left(d h x_{2}+h d x_{2}+\sigma_{2}^{\prime}+h x_{1} \lambda^{\prime}\right) \overrightarrow{h_{2}} \\
d_{f} \vec{x} & =\left[\left(\sigma_{1}^{\prime}-\sigma_{1}\right)-h x_{2}\left(\lambda^{\prime}-\lambda\right)\right] \overrightarrow{h_{1}} \\
& +\left[\left(\sigma_{2}^{\prime}-\sigma_{2}\right)+h x_{1}\left(\lambda^{\prime}-\lambda\right)\right] \overrightarrow{h_{2}}
\end{aligned}
$$

olarak elde edilir (Güngör vd. 2009).

Sonuç 2. $a_{1}=1, a_{2}=1$ ve $h=1$ olması durumunda izafi, mutlak ve sürüklenme hız vektörleri, sırasıyla, $d \vec{x}=\left(d x_{1}+\sigma_{1}-x_{2} \lambda\right) \overrightarrow{h_{1}}+\left(d x_{2}+\sigma_{2}+x_{1} \lambda\right) \overrightarrow{h_{2}}$ $d^{\prime} \vec{x}=\left(d x_{1}+\sigma_{1}^{\prime}-x_{2} \lambda^{\prime}\right) \overrightarrow{h_{1}}+\left(d x_{2}+\sigma_{2}^{\prime}+x_{1} \lambda^{\prime}\right) \vec{h}_{2}$ $d_{f} \vec{x}=\left[\left(\sigma_{1}^{\prime}-\sigma_{1}\right)-x_{2}\left(\lambda^{\prime}-\lambda\right)\right] \overrightarrow{h_{1}}$

$$
+\left[\left(\sigma_{2}^{\prime}-\sigma_{2}\right)+x_{1}\left(\lambda^{\prime}-\lambda\right)\right] \overrightarrow{h_{2}}
$$

olarak elde edilir (Müller 1963).

Sonuç 3. $h=1$ olması durumunda izafi, mutlak ve sürüklenme hız vektörleri, sırasıyla,

$$
\begin{aligned}
d \vec{x} & =\left(d x_{1}+\sigma_{1}-\frac{\sqrt{a_{1}}}{\sqrt{a_{2}}} x_{2} \lambda\right) \vec{h}_{1} \\
& +\left(d x_{2}+\sigma_{2}+\frac{\sqrt{a_{2}}}{\sqrt{a_{1}}} x_{1} \lambda\right) \overrightarrow{h_{2}} \\
d^{\prime} \vec{x} & =\left(d x_{1}+\sigma_{1}^{\prime}-\frac{\sqrt{a_{1}}}{\sqrt{a_{2}}} x_{2} \lambda^{\prime}\right) \overrightarrow{h_{1}} \\
& +\left(d x_{2}+\sigma_{2}^{\prime}+\frac{\sqrt{a_{2}}}{\sqrt{a_{1}}} x_{1} \lambda^{\prime}\right) \overrightarrow{h_{2}} \\
d_{f} \vec{x} & =\left[\left(\sigma_{1}^{\prime}-\sigma_{1}\right)-x_{2} \frac{\sqrt{a_{1}}}{\sqrt{a_{2}}}\left(\lambda^{\prime}-\lambda\right)\right] \overrightarrow{h_{1}} \\
& +\left[\left(\sigma_{2}^{\prime}-\sigma_{2}\right)+x_{1} \frac{\sqrt{a_{2}}}{\sqrt{a_{1}}}\left(\lambda^{\prime}-\lambda\right)\right] \overrightarrow{h_{2}}
\end{aligned}
$$

olarak elde edilir (Azak 2018).

Bir parametreli hareketlerde sürüklenme hiz vektörünün sıfıra eşit olduğu durumlar için tanımlanan pol noktasının koordinatlarını ifade eden aşağıdaki teoremi verelim:

Teorem 2. $E / E^{\prime}$ bir parametreli eliptik homotetik düzlem hareketinin $P$ pol noktasının koordinatları

$$
\begin{aligned}
& p_{1}=-\frac{\sqrt{a_{1}}}{\sqrt{a_{2}}} \cdot \frac{\left(\sigma_{2}^{\prime}-\sigma_{2}\right)}{h\left(\lambda^{\prime}-\lambda\right)}, \\
& p_{2}=\frac{\sqrt{a_{2}}}{\sqrt{a_{1}}} \cdot \frac{\left(\sigma_{1}^{\prime}-\sigma_{1}\right)}{h\left(\lambda^{\prime}-\lambda\right)}
\end{aligned}
$$

olarak elde edilir. Burada $\overrightarrow{B P}=\vec{p}=p_{1} \overrightarrow{h_{1}}+p_{2} \overrightarrow{h_{2}}$ olarak tanımlanmıştır. İspat: Bir parametreli eliptik homotetik harekette, hareketin pol noktaları sürüklenme hız vektörünün sıfira eşit olduğu durumlar için karakterize edilir. Bu harekette sırf dönme hareketinden kaçınmak adına $\theta \neq 0$ ve $\theta^{\prime} \neq 0$ olarak gözönüne alınacaktır. O zaman, (16) eşitliğinin sıfıra eşit olma durumu göz önüne alınırsa

$$
\begin{aligned}
& {\left[\left(\sigma_{1}^{\prime}-\sigma_{1}\right)-h x_{2} \frac{\sqrt{a_{1}}}{\sqrt{a_{2}}}\left(\lambda^{\prime}-\lambda\right)\right] \overrightarrow{h_{1}}} \\
& +\left[\left(\sigma_{2}^{\prime}-\sigma_{2}\right)+h x_{1} \frac{\sqrt{a_{2}}}{\sqrt{a_{1}}}\left(\lambda^{\prime}-\lambda\right)\right] \overrightarrow{h_{2}}=0
\end{aligned}
$$

eşitliği bulunur. $\mathrm{Bu}$ eşitlikten $E / E^{\prime}$ bir parametreli eliptik homotetik hareketin $P$ pol noktasının koordinatları

$$
\begin{aligned}
& x_{1}=p_{1}=-\frac{\sqrt{a_{1}}}{\sqrt{a_{2}}} \cdot \frac{\left(\sigma_{2}^{\prime}-\sigma_{2}\right)}{h\left(\lambda^{\prime}-\lambda\right)}, \\
& x_{2}=p_{2}=\frac{\sqrt{a_{2}}}{\sqrt{a_{1}}} \cdot \frac{\left(\sigma_{1}^{\prime}-\sigma_{1}\right)}{h\left(\lambda^{\prime}-\lambda\right)}
\end{aligned}
$$

olarak elde edilir. Burada

$\overrightarrow{B P}=\vec{p}=p_{1} \overrightarrow{h_{1}}+p_{2} \overrightarrow{h_{2}}$ olarak tanımlanmıştır. 
Sonuç 1. $a_{1}=1, a_{2}=1$ olmasi durumunda $E / E^{\prime}$ bir parametreli eliptik homotetik hareketinin pol noktaları, Öklid düzlemindeki bir parametreli homotetik hareketin pol noktalarına karşılık gelir (Güngör vd. 2009).

Sonuç 2. $a_{1}=1, a_{2}=1$ ve $h=1$ olmasi durumunda $E / E^{\prime}$ bir parametreli eliptik homotetik hareketinin pol noktaları, Öklid düzlemindeki bir parametreli düzlemsel hareketin pol noktalarına karşı1lı gelir (Müller 1963).

Sonuç 3. $h=1$ olması durumunda $E / E^{\prime}$ bir parametreli eliptik homotetik hareketinin pol noktaları, bir parametreli eliptik hareketin pol noktalarına karşılık gelir (Azak 2018).

\section{Bir Parametreli Eliptik Homotetik Düzlem Hareketi için Kanonik İzafi Sistem}

Aşağıdaki koşulları sağlayacak şekilde $\left\{B ; \vec{h}_{1}, \vec{h}_{2}\right\}$ koordinat sistemi tarafindan temsil edilen bir $E_{1}$ hareketli düzlemini seçelim:

i) Koordinat sisteminin başlangıç noktası olan $B, P$ ani dönme polü ile çakışsın.

ii) $\left\{B ; \vec{h}_{1}\right\}$ ekseni pol teğeti olsun. Yani, $(P)$ ve $\left(P^{\prime}\right)$ pol eğrilerinin ortak teğeti ile çakışsın.

Böylece i) koşulundan görebiliriz ki dönme polünün koordinatları

$p_{1}=0$ ve $p_{2}=0$

dır. Böylece Teorem 2 göz önüne alındığında $a_{1} \neq 0$ ve $a_{2} \neq 0$ için $\sigma_{1}^{\prime}=\sigma_{1}$ ve $\sigma_{2}{ }^{\prime}=\sigma_{2}$ olarak bulunur. Böylece

$$
d \vec{b}=d \vec{p}=\sigma_{1} \overrightarrow{h_{1}}+\sigma_{2} \overrightarrow{h_{2}}=d^{\prime} \vec{p}=d^{\prime} \vec{b} .
$$

sonucuna ulaşılır.

ii) koşulu göz önüne alınırsa $\sigma_{2}{ }^{\prime}=\sigma_{2}=0$ olarak elde edilir. $\mathrm{Bu}$ durumda $\sigma_{1}^{\prime}=\sigma_{1}=\sigma$ olarak seçilirse, $E_{1}$ ile gösterilen düzlem ile ilişkili $\left\{P ; \overrightarrow{h_{1}}, \overrightarrow{h_{2}}\right\}$ kanonik izafi sistemi için türev denklemleri

$$
\begin{array}{lrl}
d \overrightarrow{h_{1}}=\frac{\sqrt{a_{2}}}{\sqrt{a_{1}}} \lambda \overrightarrow{h_{2}}, & d \overrightarrow{h_{2}}=-\frac{\sqrt{a_{1}}}{\sqrt{a_{2}}} \lambda \overrightarrow{h_{1}} \\
d^{\prime} \overrightarrow{h_{1}}=\frac{\sqrt{a_{2}}}{\sqrt{a_{1}}} \lambda^{\prime} \overrightarrow{h_{2}}, & d^{\prime} \overrightarrow{h_{2}}=-\frac{\sqrt{a_{1}}}{\sqrt{a_{2}}} \lambda^{\prime} \overrightarrow{h_{1}} \\
d \vec{p}=\sigma \overrightarrow{h_{1}}, & d^{\prime} \vec{p}=\sigma \overrightarrow{h_{1}}
\end{array}
$$

halini alır.

\section{Kaynaklar}

Azak, A.Z. 2018. One-Parameter Elliptical Planar Motion. IECMSA-2018 Conference, Kyiv, Ukraine, 290-291.

Blaschke, W., Müller, H.R. 1956. Ebene Kinematik. Verlag Oldenbourg, München.

Güngör, M.A., Ersoy, S., Tosun M. 2009. Euler-Savary formula for the planar homothetic motions. Scientia Magna, 5(4), 18-25.

Hacısalihoğlu, H.H. 1971. On the Rolling of One Curve or Surface Upon Another, Proceedings of the Royal Irish Academy, 71,13-17.

Kuruoğlu, N., Tutar, A., Düldül, M. 2001. On the 1-parameter Homothetic Motions on the Complex Plane. International Journal of Applied Mathematics, 6(4), 439-447.

Masal M., Tosun, M., Pirdal, A.Z. 2010. Euler savary formula for the one parameter motions in the complex plane $C$,. International Journal of Physical Sciences, 5(01), 6-10. 
Masal, M., Ersoy, S., Güngör, M.A. 2014. Euler-Savary Formula for the Homothetic Motion in the Complex Plane $C$. Ain Shams Engineering Journal, 5, 305-308.

Müller H.R. 1963. Kinematik. Sammlung Göschen, Walter de Gruyter, Berlin.

Olcaylar, İ. 1956. Homotetik Düzlemsel Hareketler Üzerine. Doktora Tezi, Ankara Üniversitesi, Fen Fakültesi, Ankara.

Özdemir, M. 2016. An Alternative Approach to Elliptical Motion. Advances in Applied Clifford Algebras, 26, 279-304.

Tutar, A., Kuruoğlu, N., Düldül, M. 2001. On the Moving Coordinate System on the Complex Plane and Pole Points. Bulletin of Pure \& Applied Sciences, 20E(1), 1-6. 